\title{
A Hierarchical QoS Routing Protocol for the wireless sensor networks
}

\author{
Zhou Zeshun, Xu Yi, Qin Min ,Mao Chuyang, Li Layuan \\ School of Computer Science and Technology, Wuhan University of Technology, \\ Wuhan,China
}

\begin{abstract}
The large wireless sensor networks are often structured hierarchically by grouping nodes into different domains in order to deal with the scaling problem. This paper proposes a new protocol called hierarchical QoS routing protocol (HQRP) that achieves scalability by organizing the network as a hierarchy of domains using the full-mesh aggregation technique. In HQRP, each local node just only needs to maintain local routing and summary information of other domains, but does not requires any global states maintained. The HQRP uses a Reverse Best Metric Path Forwarding approach with hierarchical, topological and QoS forwarding conditions to construct the multicast tree while minimizing message overhead and satisfying delay-bandwidth and minimum energy consumption. The paper presents proof of correctness and complexity analysis of the HQRP. Simulation results show very good performance in terms of success ratio and message overhead.
\end{abstract}

\section{Keywords-Hierarchical,QoS,Routing Protocol ,WSN}

\section{INTRODUCTION}

IP multicasting is designed to enable the delivery of packets to a set of hosts that have been configured as members of a multicast group. Various protocols for IP multicast routing and these existing protocols are based on the best-effort service, so IP multicast routing with QoS guarantees on a large-scale network is required. Most of QoS-based multicast routing protocols are based on a "flat" routing model that do not scale well for large size networks. The scalability issue can be addressed by organizing the network in a hierarchy of domains.

Traditional multicast routing protocols such as DVMRP and MOSPF ${ }^{[1]}$ are unable to scale with the exponential growth of the Internet. HDVMRP ${ }^{[1]}$ divides the current flat routing region into disparate parts and assigns each a unique name. As many more routers become multicast capable, the unicast and multicast topographies in the Internet converge, and the separate routing performed by HDVMRP becomes an unnecessary burden on network bandwidth.

This paper proposes a hierarchical QoS routing protocol based on the extension of the QHMRP ${ }^{[2]}$. The proposed protocol can adapt to rapidly changing environments and works in arbitrarily large wireless sensor networks. It not only ensures fast convergence but also provides multiple guarantees for satisfying delay-bandwidth Constraints and minimum energy consumption. HQRP also allows that multicast group members can join/leave the multicast group dynamically.

The rest of this paper is organized as follows. Section2 describes the hierarchical network routing problem and model. Section 3 presents the HMRP. Section 4 gives correctness proof and complexity analysis. Simulation results are provided in Section 5. The paper concludes with Section 6.

\section{SYSTEM MODELS}

Large multicast routing domains can be decomposed into multiple sub-domains organized hierarchically, such that one sub-domain is treated as a single link in a higher level domain to scale the multicast service up to large wireless sensor networks. A domain is a set of nodes that are connected by communication links. Some nodes are connected to another domain and these nodes are called gate border nodes. We model the network and the domains as directed graphs where links can be asymmetric in both directions to make it more flexible. An example of a hierarchical network is provided in Fig.1.

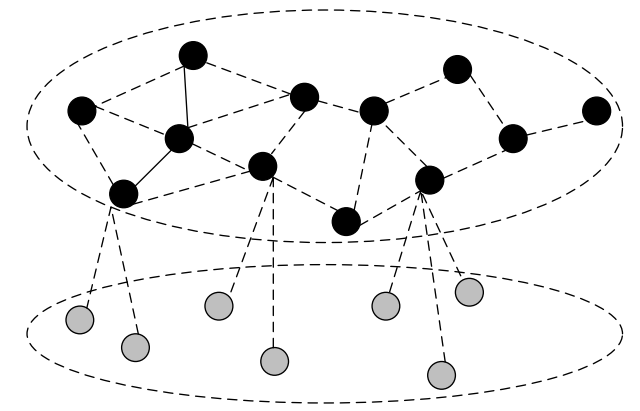

Fig.1 Hierarchical network topology

A network consists of a set of domains and links that connect them. A network is denoted as $(G, L)$ where $G=\left\{g_{\mathrm{i}} \mid g_{\mathrm{i}}=\left(V_{\mathrm{i}}, B_{i}, E_{\mathrm{i}}\right), 1 \leq i \leq G\right\}$. In order to identify a node in the network unambiguously, we use the notation $g_{\mathrm{i} *} \mathrm{v}_{\mathrm{j}}$ to refer to the node $v_{\mathrm{j}}$ of domain, $g_{\mathrm{i}}$ is the set of the links between domains. $L$ is the set of links between domains. Each inter-domain link is denoted in the same way as the physical links in a domain ${ }^{[3]}$.

A domain is modeled as a tuple $\left(V, V_{\mathrm{b}}, E\right)$, where $V$ is the set of nodes, $V_{\mathrm{b}} \subset V$ is the set of borders, and $E$ is the set of directed links among the nodes in $V$. We call those links in $E$ physical links. We denote the QoS parameter of each physical link as $(D, B)$, which means the delay of the link is $D$ units and the bandwidth is $B$ units. The delay of the physical path from $v_{0}$ to $v_{\mathrm{k}}$ is the sum of the delays of all physical links along the path, which is $\sum_{i=0}^{k-1}\left(D_{i \rightarrow i+1}\right)$. The bandwidth of it is the minimum bandwidth among all bandwidths along the physical path, which is 
$\min _{i=0}^{k-1}\left\{B_{i \rightarrow i+1}\right\}$. Energy consumption of each node is composed of emission and receiving energy, which is

$$
E(p)=\sum_{i=1}^{h} \frac{2^{2 h R_{0}-1}}{h R_{0}}\left(K_{0} d_{i}+c\right)
$$

where $h$ is the number of jumps, $d_{\mathrm{i}}$ is for the distance of the $i$ jump, $K_{0}$ is Gauss white noise, $R_{0}$ is for virtual end-to-end transmission rate., $c$ is a constant representation node receiving amount of consumption. The energy consumption of the path $P(s, m)$ is defined as sum of the energy consumption of the links on that path.In this paper, we only consider the equal bandwidth requirement defined as $B_{\mathrm{m}}$, which is decided by the group application. Suppose any on-tree node $u\left(u \in V_{T}\right)$ keeps the delay from the source $d\left(P_{T}(s, u)\right)$ along the on-tree path. When a new receiver $v$ wants to join the group, it searches a path $P(u, v)$ from any on-tree router to itself which satisfies the bandwidth and delay constraints and multicast tree energy optimization, $h(P(u, v))$ means hop counts along $P(u, v)$.

Bandwidth constraint: bandwidth $\left(\left(P_{T}(u, v)\right) \geq B_{m}\right.$

Delay constraint: delay $\left(\left(P_{T}(u, v)\right) \leq D_{m}\right.$

Energy consumption: $E(p) \rightarrow$ the least

Meanwhile the energy consumption $(T(s, M))$ should be minimum. If there are many feasible paths, the greedy algorithm selects the path which has the smallest count $h(P(u, v))$. Hence, HQRP can construct an optimal multicast tree.

\section{III.HMRP}

\section{A. Overview of HQRP}

In HQRP, we firstly establish three kinds of tables to store and maintain any node information [4], then give two definitions.

1) Link Delay Table: Full $n \cdot n$ link delay table, where $n$ is the number of the nodes in the local cluster (i.e., the cluster it belongs to). Each entry $(i, j)$ represents the delay along the link connecting node $i$ to node $j$. Note that the entries $(i, j)$ and $(j, i)$ are usually different, since the delay along the link from node $i$ to $j$ can be different from the delay of the link from node $\mathrm{j}$ to $i$.

2) Intracluster Routing Table of A Node: This table stores and maintains the optimal delay estimates and routing information along the links connecting a node to other nodes in the cluster.

3) Intercluster Routing Table: This table will store and maintain the optimal delay estimates and routing information along the links connecting a border node to other border nodes of HQRP.

In order to handle the dynamic of HQRP, The HQRP assumes that each local node measures periodically the delay along its outgoing links and forwards the cluster. Other nodes will recalculate their intracluster routing tables after receiving the update message. Similarly, each border node also checks periodically the delay along its outgoing links and forwards the information to all other border nodes in the first-level (second-level or third-level) cluster network. Other border nodes will recalculate their intercluster routing tables after receiving the intercluster updating message. The routing databases contain the main topological information that needs to be updated only when topology changes, a link /node fails, or a node joins/leaves multicast tree.

Definition 1: If a path from $u$ to $v$ can satisfy the bandwidth and delay requirement, meanwhile it has the smallest count $h(P(u, v))$. We define the path as Reverse Best Metric Path (RBMP) from $v$ to $u$.

Definition 2: RBMPF is a forwarding mode where a data packet is accepted for forwarding to other interfaces except all the incomings, if and only if it travels from a path whose reverse path is a RBMP from $v$ to $u$.

The key of RBMPF lies in how a router can identify a path traveled by a Join-request message whose reverse path is a RBMP from the new receiver to itself, Join-request messages record the route and collect the reverse route's state such as available bandwidth, accumulated delay and hops count when they travel across the network. If a node $u$ receives a Join-message, it can easily justify whether the reverse route is RMBF from the new receiver to itself or not. If passing the check, Join-messages will be forwarded towards the on-tree routers. In order to identify the RBMP, each node need to keep $D_{m}$ and $H_{o p}$ in the process of searching of a feasible path.

\section{B. Description of HQRP}

HQRP uses a receiver-initiated selection flooding algorithm in which the links that violate the bandwidth constraint will firstly be deleted, and the flooding message should keep clear of the violated links. When a new node wishes to join a multicast tree, it will send a Join-request message to border node. If The border node receiving a Join-request message is aware of the multicast tree, then it forwards the Join-request message to all the on-tree nodes or border node of the sub-domains having on-tree node. Otherwise, the border node forwards the message to another border node, then Join- request message arrives at an on-tree node, the node initiates a RBMP message. This message is flooded towards the new node by sending it to some neighbors, which in turn forwards the message to their some neighbors. To reduce message overhead during reverse selection flooding, the messages are forwarded only in those directions that satisfy certain RBMPF conditions. The forwarding conditions are selected to eliminate those messages that will not participate in establishing a feasible path between new node and the multicast tree. The related process of HQRP may be described as follows.

Switch()

Case Join-request

if (current on-tree node want to join a multicast tree) then send join-request message to its parent node else forward join-request message to all on-tree node if (current node is the highest level domain) then append the current node address to the array path

else forward the join-request message to the current border node

break

Case Join 
if $(($ multicast tree $)=$ TRUE $)$

then discard the join-request message

else reserve resources on the link to Neighbor node

if (current node address is Neighbor node)

then send Join message to Neighbor node

else if (current router is not the highest level domain)

then send updateTree message to current node's parent node if (delay $\leq D_{\mathrm{m}}$ and $\left.h(P)\right) \leq \mathrm{Hop}_{\mathrm{m}}$ )

then do the RBMPF, $h(P)=h(p)+1$

else discard Join message

break

When the new node receives a multicast tree generate message it will generate the tree and forward the multicast tree update message to its parent node. The multicast tree generate message updates the multicast tree information of the border node and is sent towards the higher level border node. Each border node should store address of all on-tree nodes within a domain and border addresses of the lower level domains that contains on-tree nodes. When a multicast tree update message arrives at a border node, the address of the node that sent the message is stored by the border node.

\section{IV.CORRECTNESS AND COMPLEXITY}

\section{A. Proof of Correctness}

Lemma 1. If changes of link delay/topology occur between time 0 and $t$, and no changes occur after time $t$, then after some finite time the routing tables (intracluster or intercluster) stored each node are correct and consistent.

Proof. For simplicity, all the updates-- intracluster updates, intercluster updates, and gate-to-node updates are assumed to be periodical. The proof is based on the induction on the number of levels of the network. Because the nature of the update protocol of the HQRP. is the flooding protocol which ensures that the packets can arrive at every node that is reachable if there is no further network topological change. Every update message sent after time $t$ will reach every node that is reachable (the delay of every packet is assumed to be bounded). Thus, all the link delay tables at each node are eventually consistent and correct even if the network is partitioned. Assume that the link delay table entries for local nodes become converged at time $t_{1}, t_{1}>t$.

Theorem 2. An optimized feasible path found by HQRP is loop-free.

Proof. HQRP has made use of RBMPF to create the optimal multicast. The paths being searched will be marked by the routing entries at the nodes. Any routing entry has a single out interface and one or multiple in interfaces Hence, the nodes will form a searching tree structure.

\section{B. Complexity Analysis}

The complexity of the HQRP is evaluated in terms of message complexity needed to construct a branch to a multicast tree for a new receiver. When the message complexity is calculated, sending a message over a link is counted as one message. Hence, for a message having traversed a path of $\mathrm{n}$ hops, $\mathrm{n}$ messages are counted. There are three steps involved in establishing a connection between a node and a multicast tree. They are unicasting a Join-request message from the node to on-tree routers, flooding messages from on-tree nodes towards the neighbor node, and sending a Join message from the neighbor node to an on-tree node. Let the time taken by the Join-request and Join messages to traverse a link including the buffering and processing time at nodes be one unit of time. Then the time taken by the Join-request and Join messages together is $\mathrm{O}(\mathrm{n} 1+\mathrm{n} 2)$, where $\mathrm{n} 1$ is the length of the path(in term of hop count) followed by the Join-request message and $n 2$ is the length of the Join message path. The worst case message overhead is $\mathrm{O}(\mathrm{n} 1+\mathrm{n} 2)$. For most cases, $\mathrm{n} 1=\mathrm{n} 2=\mathrm{n}$, hence the time is $\mathrm{O}(2 \mathrm{n})$. For bandwidth and delay requirement, the HQRP sends at most the flooding message per link for each (multicast, node) pair. The total number of flooding message is thus bounded by e, where e is the number of links in the flooding domain. Therefore, the worst case message overhead is $\mathrm{O}(\mathrm{e}+2 \mathrm{n})$.

\section{V.SIMULATION RESULT}

We use the Network Simulator $2^{[5]}$ as the basic simulation platform. Topology in Fig. 1 is generated using GT-ITM tool according to the well-known B.Waxman model ${ }^{[6]}$. The bandwidth of each link is randomly distributed in 10 100 MB, and delay of each link is randomly chosen among 10 200 ms. The performance metrics for each simulation run were calculated and the average values of performance metrics for all 100 runs were estimated.

HQRP was implemented in the simulation for flat as well as hierarchical networks on bandwidth and delay constraints. The performance metrics used to assess the performance of QHMRP are success ratio, average message overhead. These are defined as:

$$
\begin{aligned}
& \text { success ratio }=\frac{\text { number of hosts accepted }}{\text { total number of join requests }} \\
& \text { avg.meg.overhead }=\frac{\text { total number of messages sent }}{\text { total number of join requests }}
\end{aligned}
$$

The success ratio for the node delay QoS requirements are presented in Fig.2. As the same simulation parameters are used for the flat as well as hierarchical routings, the success ratio has similar behavior in both cases. There really is no feasible path exists sometimes, hence the success ratio can not reach $100 \%$. The path delay of Flooding messages increases with the node delay. Therefore, the number of messages rejected by the QoS forwarding condition increases and, hence, the success ratio decreases with an increase in the node delay. (Fig.2). 


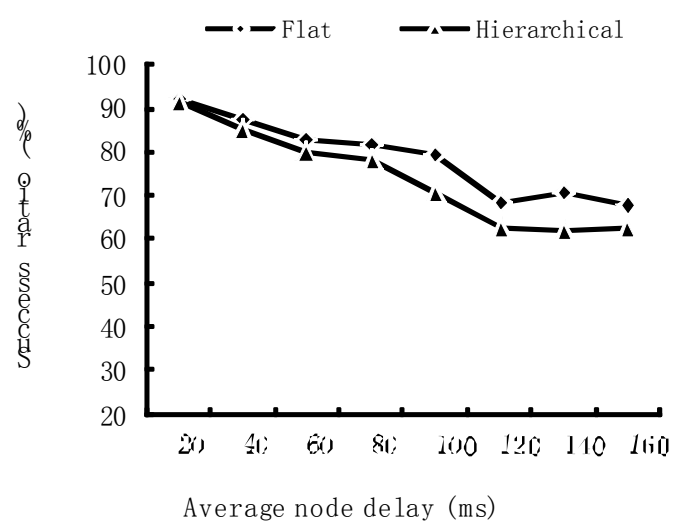

Fig.2 success ratio vs. delay

The message overhead for the delay QoS requirements are presented in Fig.3. The advantage of hierarchical routing in terms of lower message overhead as compared to the flat routing scheme can be clearly seen from the figures for both the delay and bandwidth QoS requirements. The reason lies in that when searching for all possible feasible paths, HMRP uses RBMPF to abandon Join-request messages traveling from a path whose reverse route is RBMP from the new receiver to the current node.

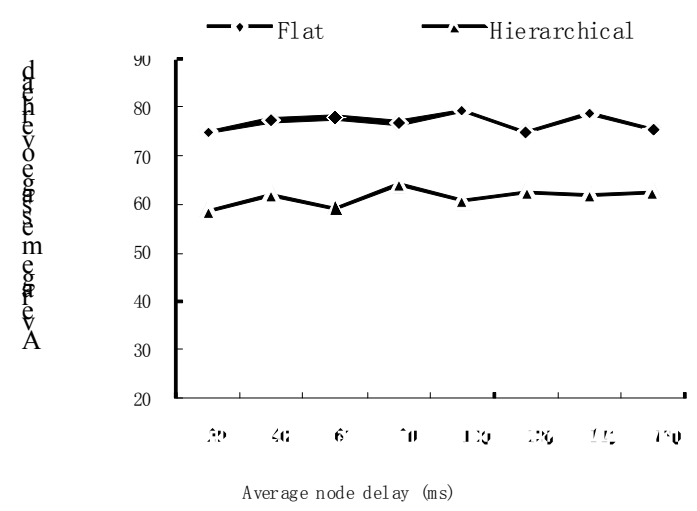

Fig. 3 average message overhead vs. delay

\section{VI.CONCLUSIONS}

HQRP can adapt dynamic topology change for dynamic large computer networks. To achieve scalability, the network is divided into domains organized into an n-level hierarchy. HQRP mainly focuses the delay and bandwidth constraints. When searching for all possible feasible paths, HQRP uses RBMPF to forward Join-request message traveling from a path whose reverse path is RBMP from the new receiver to the current node. The feasibility and performance of the protocol were assessed by simulation. Results show that the performance in terms of message overhead of the QHMRP is better than the flat routing protocol.

\section{ACKNOWLEDGMENT}

This paper is supported by National Natural Science Foundation of China (61171075) and NSF of Hubei Province (2012FFB05006,2011CDB297).

\section{REFERENCES}

[1] Shields and J. J. Garcia-Luna-Aceves. The HIP protocol for hierarchical multicast routing. in Proc. of ACM PODC'98, June 1998, pp. 257-266.

[2] S. Pradhan, QoS-Aware Hierarchical Multicast Routing on Next Generation Internetworks, M.Sc. Thesis, Department of Computer Science, University of Manitoba, Winnipeg, Canada, 2000.

[3] S. Chen K.-S. Lui, K. Nahrstedt. Hierarchical QoS Routing in Delay-Bandwidth Sensitive Networks. In IEEE LCN, Tampa, FL, USA, 2000.

[4] W. T. Tsai, C. V. Ramamoorthy, W. K. Tsai, and O Nishiguchi. An adaptive hierarchical routing protocol. IEEE TRANSACTIONS ON COMPUTERS, VOL 38. NO. 8. AUGUST 1989.pp.1059-1074

[5] ns-2. http://www.isi.edu/nsnam/ns

[6] M. Waxman. Routing of multipoint connections. IEEE Journal on Selected Areas in Communications, September 1988,14:1617-1622. 